\title{
Let's Repair Peripheral Arterial Disease PAD-affected Blood Vessels by Distal Vein Arterialization
}

\author{
Tomiyasu Koyama ${ }^{1 *}$, Tadahiro Sasajima ${ }^{2}$ and Shinsuke Kikuchi ${ }^{2}$ \\ ${ }^{1 * H o k k a i d o ~ U n i v e r s i t y, ~ J a p a n ~}$ \\ ${ }^{2}$ Asahikawa Med. University and Edogawa Hospital, Japan \\ *Correspondence to: Tomiyasu koyama, Hokkaido University, Japan; E-mail: tomkoyamajp@yahoo.co.jp
}

Received: December 09, 2017; Accepted: December 19, 2017; Published: December 27, 2017;

\section{Short communication}

One human foot is surgically cut off each 30 seconds in the world because of the artery destruction by peripheral artery disease (PAD) in the world [1]. To rescue PAD-affected ischemic feet, arterial blood should be retrogradely introduced into venous blood vessels in the foot. But it may be difficult for readers to accept such a strange statement, since oxygen is normally supplied only by the arterial blood flowing through arterial micro-vessels.

However, since in PAD patients their arterial system is destroyed by the disease, blood cannot flow into the arterial micro-vessel system in the foot and fails to supply oxygen to foot muscles. On the other hand, veins remain still open in PAD-affected patients. It can repeat cell recruitment and branching, anastomosing to reform the oxygen supply route fine venular networks and effective in reforming as oxygen supply route for surrounding muscle tissues. The venous system has the capacity to rearrange the oxygen supply route.

1. Blood flow and Oxygen can be transported to the living tissues via venous route as suggested by a thermal camera
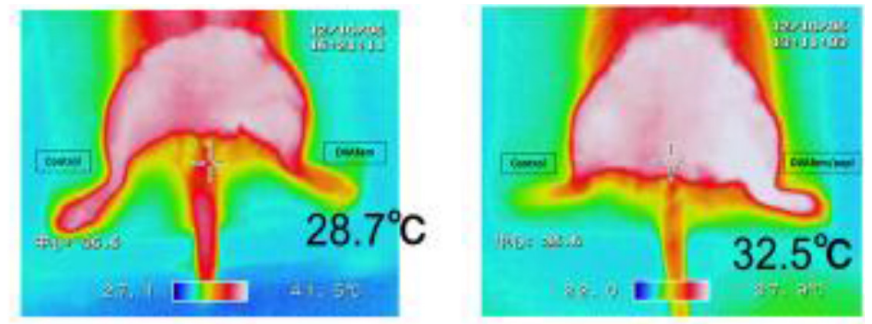

A

B

Figure1. Examples of thermo-camera recordings obtained in the control A and DVA feet B.

A: The right femoral artery and vein remained untouched, $30.6^{\circ} \mathrm{C}$, while the left femoral artery was ligated in the left foot, $28.7^{\circ} \mathrm{C}$ for control. The left femoral artery was ligated but the left untouched.

B: Venous valves in the left femoral vein were destroyed. The skin temperature on the right foot was $24.6{ }^{\circ} \mathrm{C}$. The femoral artery blood was forced to flow retrograde through the left femoral vein. The white colouring of the left foot indicated the rise in the skin temperature to $32.5^{\circ} \mathrm{C}$ i.e. the increase in the local blood flow.
More oxygen is brought to the left foot tissue by the retrograde blood flow.

2. How to anastomose the femoral artery and vein in rats
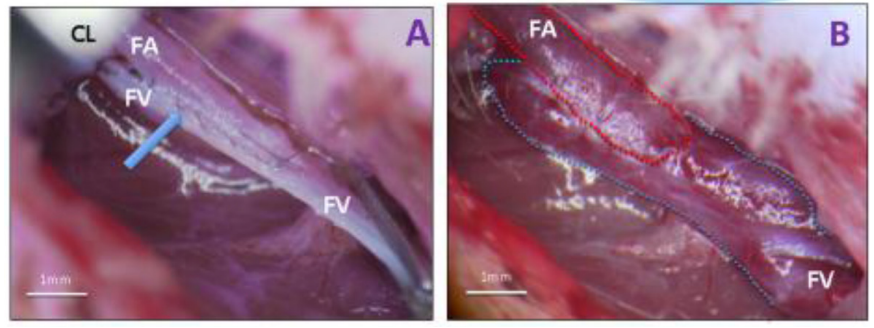

Figure 2. After destruction of the venous valves the incision in the femoral vein and artery were opposed in a side-to-side manner and the vessels stitched together under microscopic control, using 11-0 monofilament nylon in a continuous running suture. The vein was closed in the proximal position. This procedure formed an opening from artery to vein. The removal of the clamps caused an immediate flow of arterial blood into the femoral vein and its dilation (quick warming of the skin). The surrounding skin became pink

3. A cine angiographic confirmation of the no inflow of the arterial blood to the vein with intact femoro/popliteal valves and the inflow to the periphery with destructed valves in the left hind limb. The arterio-venous anastomosis formation and valve destruction were made.

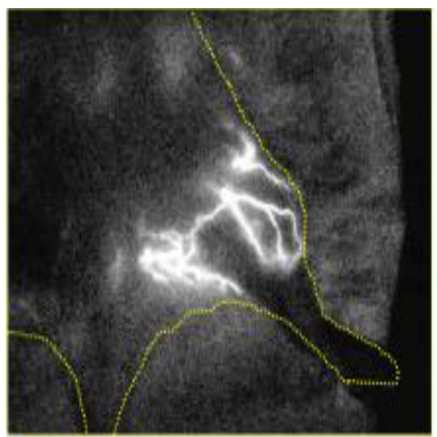

Valves intact

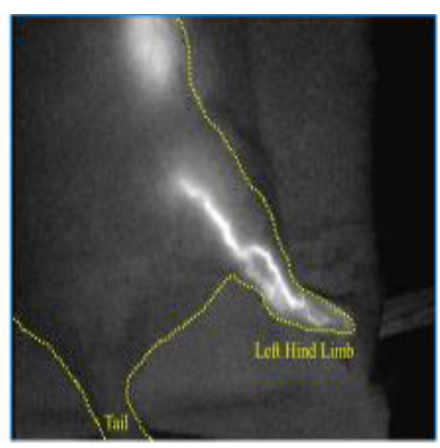

Valves destructed
Figure 3. A cine angiographic confirmation of the no inflow of the arterial blood to the vein with intact femoro/popliteal valves and the inflow to the periphery with destructed valves in the left hind limb. The arterio-venous anastomosis formation and valve destruction were made. 
4. What is the final result? Recovery of the foot!

An example of a rescued foot and re-established micro vascular networks in a human PAD patient. (Cited from Sasajima et al. $[2-5]$

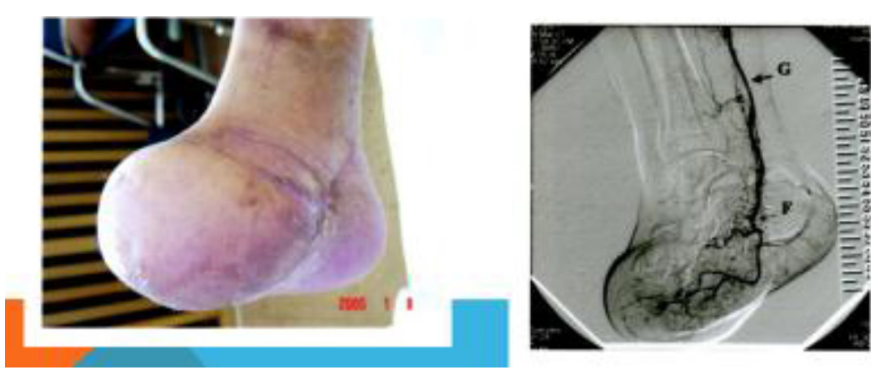

Figure 4. An example of a rescued foot and re-established micro vascular networks in the foot of a human PAD patient and the angiographic confirmation of the blood flow into the re-established vascular network (Sasajima et al.). A branch of the tibial artery was grafted to a branch of saphenous vein. The re-established arterial net could be confirmed.

The wound was covered with the healthy skin flap prepared on the patient's healthy abdominal skin. New blood vessels grew up from the vascular endothelial cells. Probably endothelial growth factor released from the fresh skin flap stimulated the growth of new micro-vessels and vascular growth factor for foot skin and muscle.

Calculation of the diffusion front in the venular network [6, 7] suggested that the oxygen diffusion front covered the tissue cylinder.

\section{References}

1. Boulton AJ, Vileikyte L, Ragnarson-Tennvall G, Apelqvist J (2005) The global burden of diabetic foot disease. Lancet 366: 1719-1724. [crossref]

2. Sasajima T, Azuma N, Uchida H, et al. (2010) Combined distal venous arterialization and free flap for patients with extensive tissue loss. Annals of Vascular Surgery 24: $373-81$

3. Koyama T, Sasajima T, Kikuchi S (2016) Oxygen transport to diseased hind limb: A comparison of medical treatments. Medical Research Archives 4: 2-16.

4. Sasajima T, Koyama T2 (2013) Biological maintenance of distal vein arterialization. Adv Exp Med Biol 765: 245-250. [crossref]

5. Koyama T, Sasajima T (2012) Retrograde perfusion of the hind leg in diabetic patients suffering from arteriosclerotic obliterance: theoretical considerations of oxygen. Adv Exp Med Biol 737: 259-262.

6. Engelson ET, Schmid-Schönbein GW, Zweifach BW (1985) The microvasculature in skeletal muscle. III. Venous network anatomy in normotensive and spontaneously hypertensive rats. Int J Microcirc Clin Exp 4: 229-248. [crossref]

7. Koyama T, Sugihara-Seki M, Sasajima T, Kikuchi S (2014) Venular valves and retrograde perfusion. Adv Exp Med Biol 812: 317-323. [crossref]

Citation:

Tomiyasu Koyama, Tadahiro Sasajima and Shinsuke Kikuchi (2018) Let's Repair Peripheral Arterial Disease PAD-affected Blood Vessels by Distal Vein Arterialization. Cardiac Sci Res J Volume 1(1): 1-2 